\title{
Endoscopic transnasal approach for resection of locally aggressive tumors in the orbit
}

\author{
Masahiro Shin, MD, ${ }^{1}$ Kenji Kondo, MD, ${ }^{2}$ Shunya Hanakita, MD, ${ }^{1}$ Keigo Suzukawa, MD, ${ }^{2}$ \\ Taichi Kin, MD, ${ }^{1}$ Masaaki Shojima, MD, ${ }^{1}$ Daichi Nakagawa, MD, ${ }^{1}$ and Nobuhito Saito, MD1 \\ Departments of ${ }^{1}$ Neurosurgery and ${ }^{2}$ Otolaryngology, The University of Tokyo Hospital, Tokyo, Japan
}

\begin{abstract}
OBJECT In recent years, application of endoscopic transnasal surgery (ETS) has been expanded to orbital lesions, and preliminary results have started to be published for medially located soft mass lesions. However, reports on experience with endoscopic intraorbital surgery aimed at resection of invasive skull base tumors remains quite limited. This report presents the authors' experience with ETS for locally aggressive tumors involving the orbit.
\end{abstract}

METHODS ETS was performed for 15 cases of aggressive tumors involving the orbit: 5 meningiomas (meningothelial, $n$ $=3$; atypical, $n=1$; anaplastic, $n=1$ ), 4 chordomas, 2 chondrosarcomas, and 4 others (metastasis from systemic myxofibrosarcoma, schwannoma, inverted papilloma, and acinic cell carcinoma, $n=1$ each). Among these, 9 tumors were located outside the periorbita and 6 inside the periorbita. In 6 intraperiosteal tumors, 5 were intraconal lesions, of which 3 arose in the muscle cone (anaplastic meningioma, optic sheath meningioma, and metastatic myxofibrosarcoma), and 2 meningothelial meningioma had invaded from the sphenoid ridge or the cavernous sinus into the muscle cone through the optic canal and the superior orbital fissure. A case of schwannoma originated around the cavernous sinus and pterygopalatine fossa and extended extraconally into the periorbita. Intraoperatively, ethmoid air cells and the lamina papyracea were removed, and extraperiosteal tumors were safely approached. For intraperiosteal tumors, the periorbita was widely opened, and the tumors were approached through the surgical window between the rectus and oblique muscles.

RESULTS Gross-total resection was achieved for 12 of the 15 tumors, including 2 intraconal lesions. After surgery, exophthalmos resolved in all 8 patients with this symptom, and diplopia resolved in 5 of 6 patients. Improvement of visual symptoms was reported by 4 of 5 patients with loss of visual acuity or constriction of the visual field. Postoperatively, 1 patient showed mild, transient worsening of existing facial dysesthesia, and another showed transient ptosis and mild hypesthesia of the forehead on the affected side. All those symptoms resolved within 3 months. No patients showed enophthalmos, worsening of diplopia or visual function, or impairment of olfaction after surgery.

CONCLUSIONS ETS appears acceptable as a less-invasive alternative for treating aggressive tumors involving the orbit. For extraperiosteal tumors, gross-total removal can generally be achieved without neurological complications. For intraperiosteal tumors, surgical indications should be carefully discussed, considering the relationship between the tumor and normal anatomy. Wide opening of the periorbital window is advocated to create a sufficient surgical pathway between the extraocular muscles, allowing a balance between functional preservation and successful tumor resection.

http://thejns.org/doi/abs/10.3171/2014.11.JNS141921

KEY WORDS chordoma; endoscopic transnasal surgery; medial orbitotomy; meningioma; orbit; orbital tumor; oncology

$\mathrm{S}$ INCE the introduction of endoscopic technology into the field of neurosurgery, the role of the endoscopic transnasal approach to skull base tumors has dramatically expanded. Presently, various types of tumors can be safely approached through the nostril, and endoscopic approaches are accepted as one of the least-invasive alternatives to conventional transcranial approaches. ${ }^{5,8,9,12,21,22,25,26}$ In recent years, these techniques have been expanded to approach orbital lesions, and preliminary results have started to be published for well-demarcated and medially located lesions. ${ }^{6,13,17,18,19}$ In these reports, the main purpose of the surgery is biopsy or resection of small, benign softtissue lesions such as hemangiomas, but reports on experience with endoscopic intraorbital surgery aimed at resection of invasive skull base tumors remain quite limited. ${ }^{18}$

We present herein our experience with the endoscopic

ABBREVIATION ETS = endoscopic transnasal surgery.

SUBMITTED August 19, 2014. ACCEPTED November 5, 2014.

INCLUDE WHEN CITING Published online May 22, 2015; DOI: 10.3171/2014.11.JNS141921.

DISCLOSURE The authors report no conflict of interest concerning the materials or methods used in this study or the findings specified in this paper. 


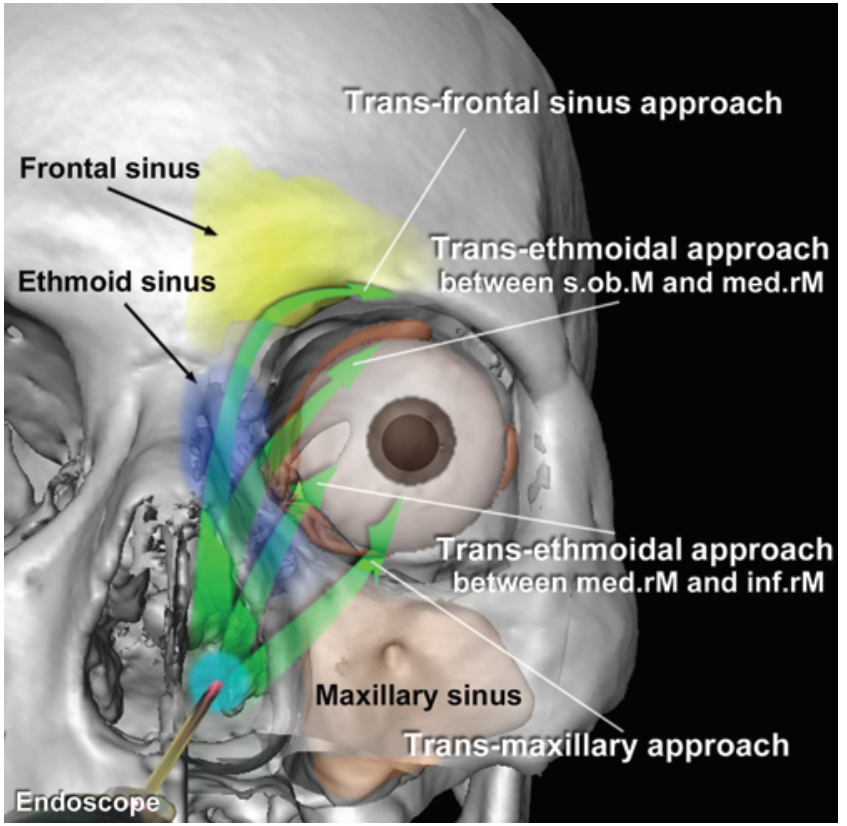

FIG. 1. Schematic of the transnasal route to the orbit (green arrows). Intraorbital tumors are approached through the frontal, ethmoid, and maxillary sinuses. For intraconal lesions, opening the periorbita reveals 3 intraorbital muscles (superior oblique muscle [s.ob.M], medial rectus muscle [med.rM], and inferior rectus muscle, [inf.rM]), and the tumor is approached between them.

transnasal approach for invasive skull base tumors involving the orbit. Surgical indications and possible advantages of this relatively new approach for locally aggressive skull base tumors involving the orbit are discussed.

\section{Methods}

\section{Patients}

The indication for a transnasal approach to an orbital tumor is a lesion located close to the medial aspect of the orbit or in the orbital apex. For intraconal tumors, this technique is recommended when a sufficient approach route can be expected between the rectus muscles or between the medial rectus and superior oblique muscles as determined by radiographic imaging (Figs. 1 and 2).

Since 2010, we have performed endoscopic transnasal surgery (ETS) for 15 skull base tumors involving the orbit (Table 1), comprising 5 meningiomas (meningothelial, $\mathrm{n}=3$; atypical, $\mathrm{n}=1$; anaplastic, $\mathrm{n}=1$ ), 4 chordomas, 2 chondrosarcomas in the paranasal sinus, and 4 others (metastasis from systemic myxofibrosarcoma, trigeminal schwannoma, inverted papilloma, and acinic cell carcinoma, $\mathrm{n}=1$ each).

Among these, 6 tumors were located in the periorbita. Of these 6 tumors, 5 were situated intraconally, of which 3 had arisen within the muscle cone (anaplastic meningioma, optic sheath meningioma, and metastatic myxofibrosarcoma, $\mathrm{n}=1$ each), and 2 meningothelial meningiomas had invaded from the sphenoid ridge or the cavernous sinus into the muscle cone through the optic canal and the superior orbital fissure. One case of schwannoma had originated around the cavernous sinus and pterygopalatine fossa, extending extraconally into the periorbita.

Two chordomas had recurred within the orbital apex and were wedged between the optic sheath and superior orbital fissure, attaching to the internal carotid artery outside the dural membrane and extending into the orbit along the surface of the periorbita. In the remaining 7 patients, the tumor had arisen from the ventral skull base region (chordomas and chondrosarcomas, $\mathrm{n}=2$ each; atypical meningioma, inverted papilloma, and acinic cell carcinoma, $\mathrm{n}=1$ each), partially destroying the orbital bone and invading the orbit outside the periorbita.

Ten patients, some with multiple symptoms, showed rapid progress of symptoms, with 8 presenting with exophthalmos, 6 with diplopia, and 5 with loss of visual acuity or constriction of the visual field. Two patients with chordomas of the orbital apex showed no neurological symptoms, but the tumors revealed apparent growth on follow-up radiographic imaging, and endoscopic surgery was applied (Table 1).
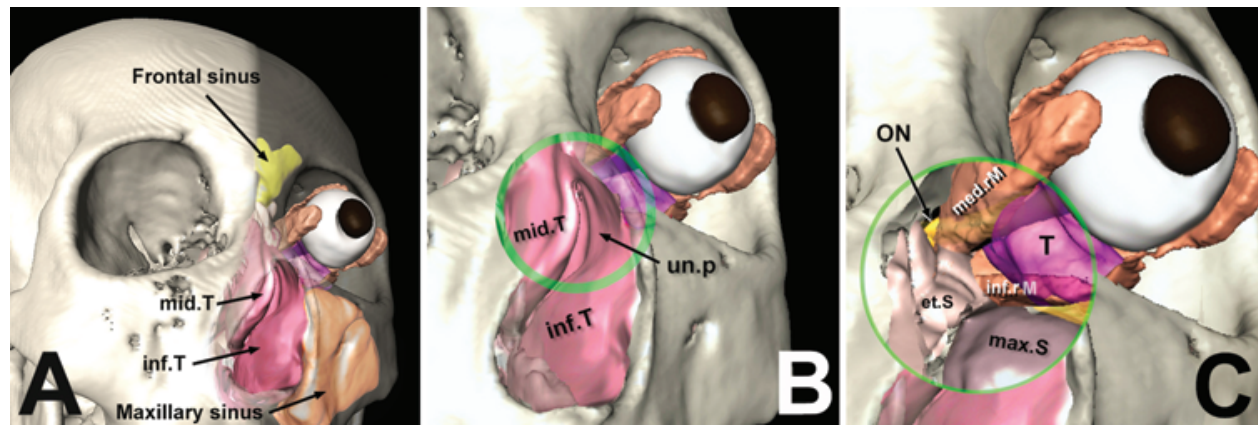

FIG. 2. Schematic illustrations of the surgical approach for the intraconal tumor in the left orbit. The left nasal cavity is observed from the outside in the same direction as the lateral-upward view with the $30^{\circ}$ endoscope. A: The middle nasal turbinate (mid.T) and inferior nasal turbinate (inf.T) are disclosed to show the anatomy on the lateral wall of the unilateral nasal cavity (the cartilaginous septum is not shown on the image). B: Endoscopic view from inside the left nasal cavity (green circle) reveals the middle nasal turbinate and the uncinate process (un.p). C: After removal of the middle nasal turbinate and the uncinate process, the ethmoid air cells (et.S) and the maxillary sinus (max.S) are opened, and the medial orbital wall is exposed. Following removal of the lamina papyracea, the periorbita is widely opened, and the intraconal tumor $(T)$ was exposed between the medial rectus muscle (med.rM) and the inferior rectus muscle (inf.rM) (as shown in C). ON = optic nerve. 


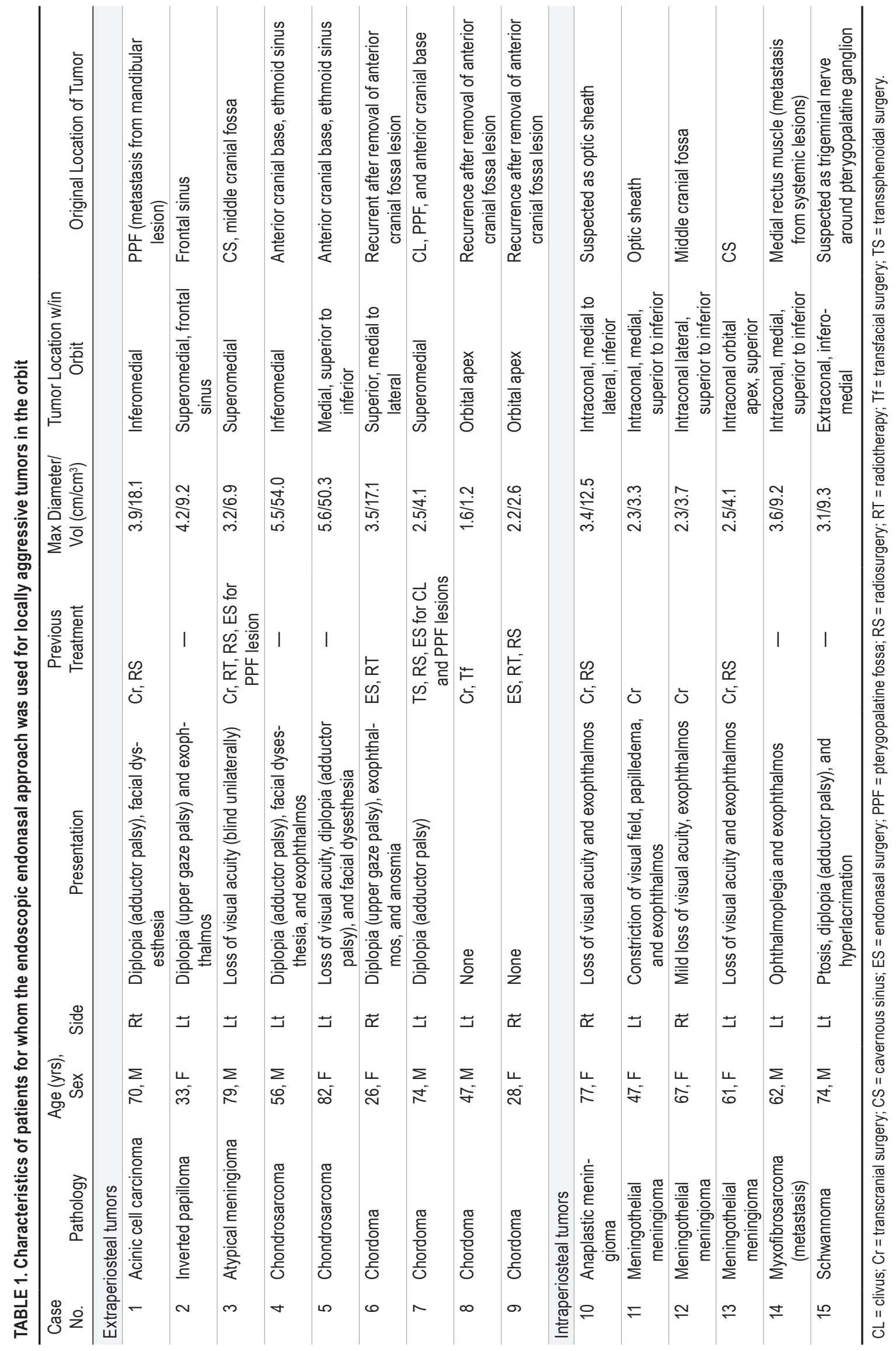


In all cases, patency of the ophthalmic artery was verified using CT or MR angiography before surgery. In 1 patient with extraperiosteal tumor, origin of the ophthalmic artery was not found on the internal carotid artery, and cerebral angiography confirmed that retinal blood flow was supplied by the meningeal artery. All 6 patients with intraperiosteal lesions underwent cerebral angiography to verify routes of ophthalmic arteries and central retinal arteries.

\section{Surgical Procedures}

\section{Surgical Equipment}

Endoscopes $4 \mathrm{~mm}$ in diameter with $0^{\circ}$ and $30^{\circ}$ lenses were mainly used (rigid scopes, 175-180 mm long; Karl Storz Endoscopy Japan), and $70^{\circ}$ lenses were used for removal of the superolateral lesions, lesions in the retrobulbar space, or superficial lesions in the frontal sinus or close to the superior palpebral. A suction tube with an irrigating function is essential to clear the endoscopic lens when clouded by mucus or blood. Narrow-shaft, bipolar coagulators and various dissecting or curetting devices were used simultaneously. A high-frequency radiosurgical device with a sharp malleable tip (Surgitron, Ellman International) was used to cut or remove the nasal mucosa. To remove the bony structure on the orbital wall or paranasal sinus, a drill system (Midas Rex, Medtronic) with a diamond bur on a long and slightly curved shaft was used. Also, an XPS microdebrider (Medtronic) proved very useful to swiftly remove the tumor and prevent surgical dissemination, which may accompany piecemeal tumor resection. To reach the orbital regions, the tips of all instruments (ringcurette, forceps, suction with irrigating function, and bipolar coagulators) are designed to be malleable, able to be curved in various degrees. The endoscope was stabilized in the surgical field with a Point Setter robotic holding device (Mitaka Kohki) and routinely equipped with the Stealth Station neuronavigation system (Medtronic).

\section{Approach to the Orbit}

The patient was placed in the supine position, with the head raised $10^{\circ}$. The head was fixed with a Mayfield 3 -point head holder and slightly rotated toward the operator in a chin-up position. After the nasal cavities were sterilized, nasal pledgets soaked with $0.02 \%$ epinephrine solution were placed on the nasal mucosa for mucosal decongestion. Monitoring of cranial nerve function (visual evoked potential monitoring with 16 red high-luminosity light-emitting diodes and electromyography of the extraocular muscles [Unique Medical]) appears useful to avoid postoperative cranial nerve deficit). ${ }^{3,10,23,24}$ The patient was draped in sterile fashion, exposing the nose and eye on the affected side to confirm the level of exophthalmos or enophthalmos intraoperatively after tumor removal.

Nasal pledgets are removed immediately before the start of surgery. We approach through a single nostril (Fig. 2A). To retain sufficient surgical space in the orbital region, the ethmoid air cells and middle nasal turbinate on the approach side are removed (Fig. 2B and C). In recent cases, the medial surface of the nasal mucosa on the middle turbinate has been preserved and used to cover the mucosal defect on the medial surface of the orbit.
Observed from a nostril, the orbit is located rostrolaterally, separated from the nasal cavity by the ethmoid sinus on the medial aspect and from the maxillary sinus by the floor. The infraorbital nerve runs in the rostrolateral corner of the maxillary sinus. The orbital roof consists of the bottom of the frontal sinus in the anteromedial part and of the anterior cranial base in the posterior part (Fig. 1).

When the tumor is located in the superomedial aspect of the orbit, removal of the ethmoid air cells is extended to the frontal base, and the anterior ethmoid artery is exposed and resected to facilitate the approach (Figs. 3 and 4). If the tumor is extended more anteriorly, the natural ostium of the frontal sinus is enlarged, and the lesion is approached through the frontal and ethmoid sinuses (Fig. 4). When the tumor is located in the inferomedial aspect, the lateral wall of the nasal cavity is removed around the uncinate process to enter the maxillary sinus, and the orbital bone is removed medial to the route of the infraorbital nerve. The lamina papyracea is exposed and carefully removed, and the periorbita is then widely disclosed (Figs. 5-7). To approach the orbital apex, the posterior part of the lamina papyracea is initially removed, and bony removal is extended posteriorly along the optic canal using a micro-Kerrison punch. The tumor location is finally confirmed with navigation.

For the approach to intraperiosteal lesions, the periorbita is incised and widely opened with a microknife and scissors to disclose the intraorbital fat, orbital muscles, and tumor. After opening the periorbita, the thin layer of intraorbital fat bulges out. Removal of the fat and redundant tissues reveals 3 intraorbital muscles (superior oblique muscle, medial rectus muscle, and inferior rectus muscle), and the tumor is approached while retracting the muscles (Figs. 5 and 6). Relatively large tumors are sometimes exposed between these muscles, and dissection of the tumor margins is not difficult. Because the motor nerves, which innervate the extraocular muscles, go through the orbital apex into the middle part of the inner muscle belly, dissection of the tumor capsule from the inner surface of the extraocular muscles is started at the anterior margin of the tumor and proceeds in a posterior direction along the routes of the nerves and vessels. This procedure should be accompanied by careful monitoring by electromyography (Figs. 5 and 6). For tumors extending to the retrobulbar space, reaching parts behind the orbit is sometimes very difficult. For those cases, we use the "eyeball compression technique." Briefly, the eyeball on the affected side is gently compressed against the upper palpebra from the outside, thus loosening the muscles to allow sufficient space between the extraocular muscles and simultaneously pushing the tumor component behind the eyeball, out of the periorbital window (Fig. 7). This procedure demands careful attention to the patient's heart rate, considering the risk of stimulating the vagal nerve reflex. After tumor removal, meticulous hemostasis is obtained with bipolar cautery or placement of hemostatic materials.

\section{Reconstruction of the Periorbita}

After removal of the intraperiosteal tumor, the fascia lata and fat tissue are harvested and used for reconstruction of the periorbital defect. The fascia lata is placed to cover the periorbital defect in 2 layers, inside and outside 

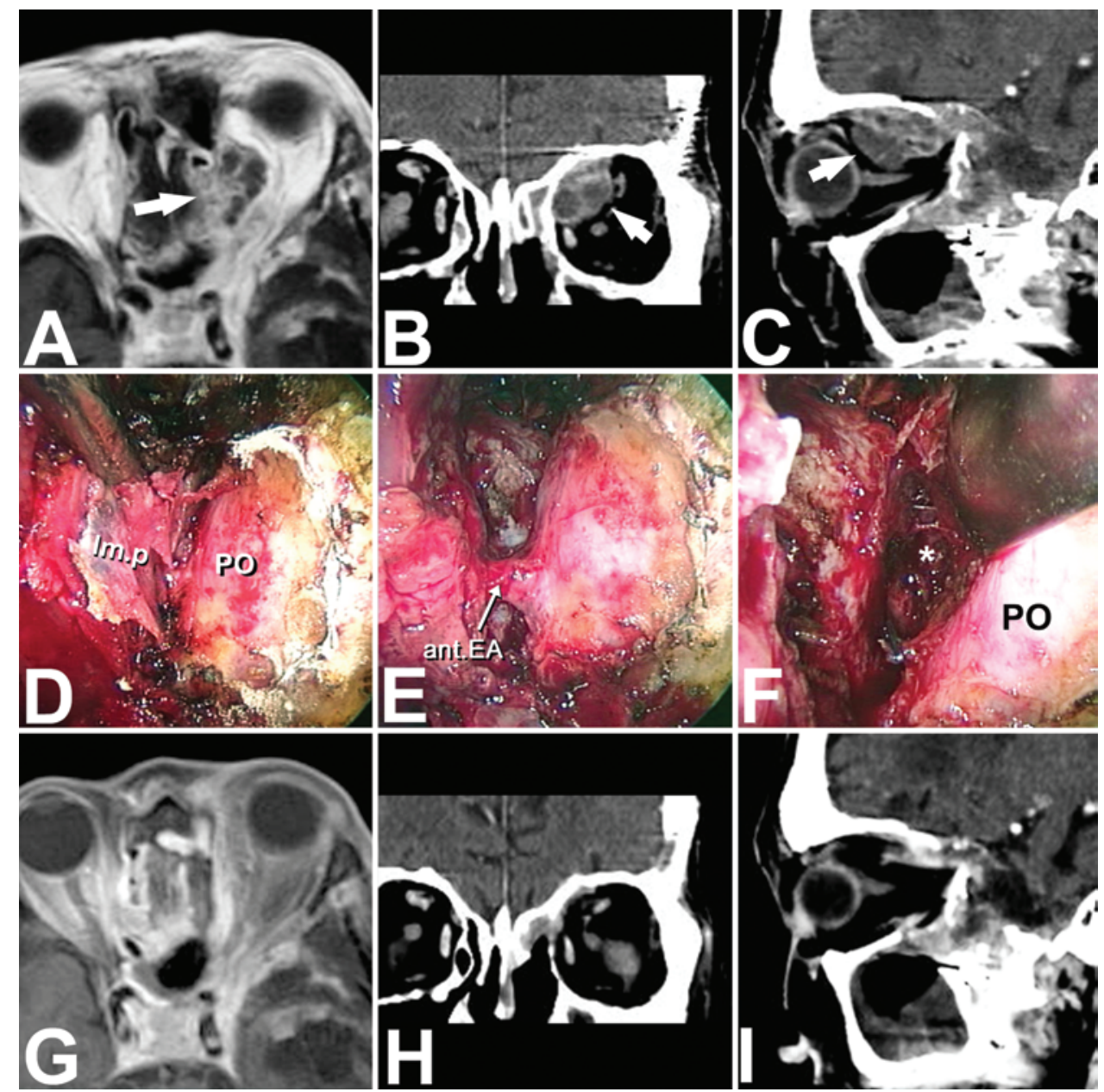

FIG. 3. A case of recurrent atypical meningioma invading the orbit (Case 3 in Table 1). Preoperative images show the tumor on the superomedial side of the right orbit (arrows in A-C). Axial T1-weighted Gd-enhanced MR image discloses a heterogeneously enhancing lesion outside the periorbita (A). CT scans with contrast enhancement show that the orbital lesion has invaded from the sphenoid ridge (B, coronal section; $\mathbf{C}$, sagittal section). After removal of the ethmoid air cells, the lamina papylacea (Im.p) is opened, and the medial periorbita (PO) is exposed (D). When the medial orbitotomy is extended posteriorly, the anterior ethmoidal artery (ant.EA) is observed, coagulated, and sectioned to facilitate further retraction of the periorbita $(E)$. With gentle retraction of the periorbita, the rostral part of the orbit is exposed and the tumor (asterisk) is removed $(F)$. Postoperative images show successful removal of the orbital lesion (G, axial T1-weighted MR image with Gd enhancement; $\mathbf{H}$, coronal CT scan with enhancement; I, sagittal CT scan with enhancement).

the periorbita. The inlay fascia is inserted into the periorbital defect to prevent ocular muscles from bulging out of the defect, while overlay fascia is placed to broadly cover the inlay fascia and exposed periorbital margins (Figs. 5 and 6). A sufficiently preserved medial surface of the nasal mucosa on the middle turbinate can replace the overlay fascia. These fascia or nasal mucosa are fixed with fibrin glue and mildly compressed with a balloon placed in the upper nasal cavity. The quantity of the implanted fat graft and inflation of the balloon are adjusted by the position of the eyeball as observed externally.

After reconstruction of the skull base floor, nasal packing with chitin-coated gauze is placed unilaterally for 36 hours. A nasal balloon is placed for 5 days, and every patient undergoes a check-up by the ophthalmologist and an endoscopic examination by the otolaryngologist at 1 week postoperatively.
Patients visit our outpatient clinic at 1 and 3 months postoperatively for neurological evaluation and a second endoscopic rhinologic check-up.

\section{Results}

Among the 15 tumors, gross-total resection was achieved in 12, including 2 intraconal lesions (1 anaplastic meningioma, 1 metastatic tumor; Table 2). One patient with intraconal meningioma showed rapid progression of the residual tumor after previous transcranial resection followed by radiosurgery (Case 10). The pathology, identified 10 years earlier, had been benign meningothelial meningioma, and we performed gross-total removal while successfully preserving residual vision and ocular movements (Fig. 5). However, histopathological examination of the resected specimen revealed that the tumor had advanced to become anaplastic. Despite adjuvant fraction- 

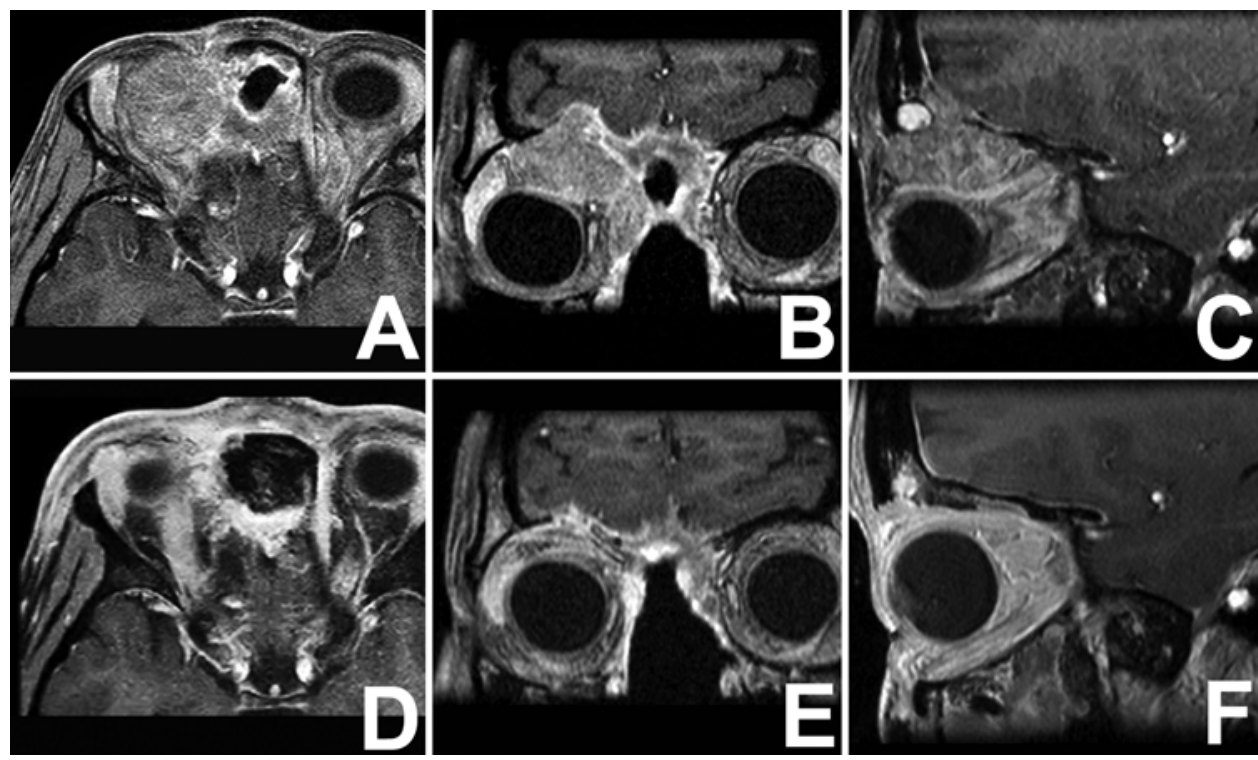

FIG. 4. Case 6. A case of large recurrent chordoma with extensive invasion into the right orbit and frontal sinus. At an outside hospital, the nasal septum had been completely removed at partial tumor resection. After failure of conventional radiotherapy, the patient was referred to our hospital. Preoperative Gd-enhanced T1-weighted MR images show the tumor strongly compressing the eyeball outside the periorbita in an inferolateral direction (A, axial; $\mathbf{B}$, coronal; $\mathbf{C}$, sagittal), and the patient presented with exophthalmos and upper gaze palsy. After ETS, MR images show complete removal of the tumor (D, axial; $\mathbf{E}$, coronal; $\mathbf{F}$, sagittal), and her symptoms completely resolved.

ated radiotherapy with 60 Gy for prophylaxis, the tumor had recurred by 1 year later. We confirmed by angiography that blood flow to the eyeball was mainly supplied from the facial artery and were convinced that eyeball necrosis would be less likely to occur in association with removal of all intraorbital components beside the eyeball. ${ }^{11}$ At this time, at which point vision and ocular movements were completely impaired, we decided to remove the tumor with adjacent orbital anatomies except for the eyeball in a second surgery (Fig. 8).

In 3 patients with intraconal meningioma, postoperative MRI revealed residual tumors in the superolateral part of the orbit or orbital apex (Cases 11-13). In 1 case of optic sheath meningioma (Case 11; Fig. 6), more than $40 \%$ deterioration of visual evoked potentials continued during surgery when dissecting the tumor but finally resolved at the end of the surgical procedure. In another case (Case 13), electromyography of the extraocular muscles frequently showed transient injury patterns to vertical movements during dissection of the tumor from the levator palpebrae muscle and superior rectal muscle. In those 2 cases, the results of intraoperative monitoring compelled us to suspend further resection, and the surgeries resulted in partial resection. In one patient (Case 12), the minimum necessary extent of the lamina papyracea was removed, and the tumor was approached through the small periorbital window. With resection of the inferomedial parts of the tumor, we could not retain sufficient space to displace the extraocular muscles and failed to reach the superolateral part.

After surgery, exophthalmos resolved in all 8 patients, and diplopia resolved in 5 of 6 patients. Four of 5 patients described improvement of visual symptoms (Cases 5, 10, 11, and 13). Of these, loss of visual function in 1 patient
(Case 10) had resulted from previous transcranial surgery, and relapse of the tumor gradually aggravated this symptom. After gross-total resection of the intraconal tumor in the first transnasal surgery, the patient subjectively reported improvement of vision, although this could not be confirmed on postoperative ophthalmological examination. In 2 patients with intraconal meningioma (Cases 11 and 13), surgery resulted in partial resection, but ophthalmological examination after surgery showed improvement of the visual field, papilledema (Case 11), and visual acuity (Case 13).

In a patient with metastatic myxofibrosarcoma (Case 14; Fig. 7), the tumor showed rapid enlargement with progressive exophthalmos, but the effectiveness of radio- and chemotherapy for aggressive myxofibrosarcoma remains controversial. ${ }^{14}$ Intraoperative examination disclosed tumor invasion into the medial rectus muscle, and we decided to perform total resection of the tumor with the adjacent part of the medial rectus muscle. After surgery, exophthalmos was completely resolved, but diplopia showed no improvement.

Regarding complications, 1 patient showed mild, transient worsening of existing facial dysesthesia (Case 4), and another showed transient ptosis and mild hypesthesia of the forehead on the affected side, along with dissection of the tumor from the levator palpebral muscle and frontal nerve (Case 13). All those symptoms resolved within 3 months. No patients showed enophthalmos, worsening of diplopia or visual function, or impairment of olfaction after surgery.

\section{Discussion}

In this article, we have presented our experience with ETS for locally aggressive tumors involving the orbit. Such tumors can be divided into 2 categories by their anatomical location: whether the tumor is located outside the 

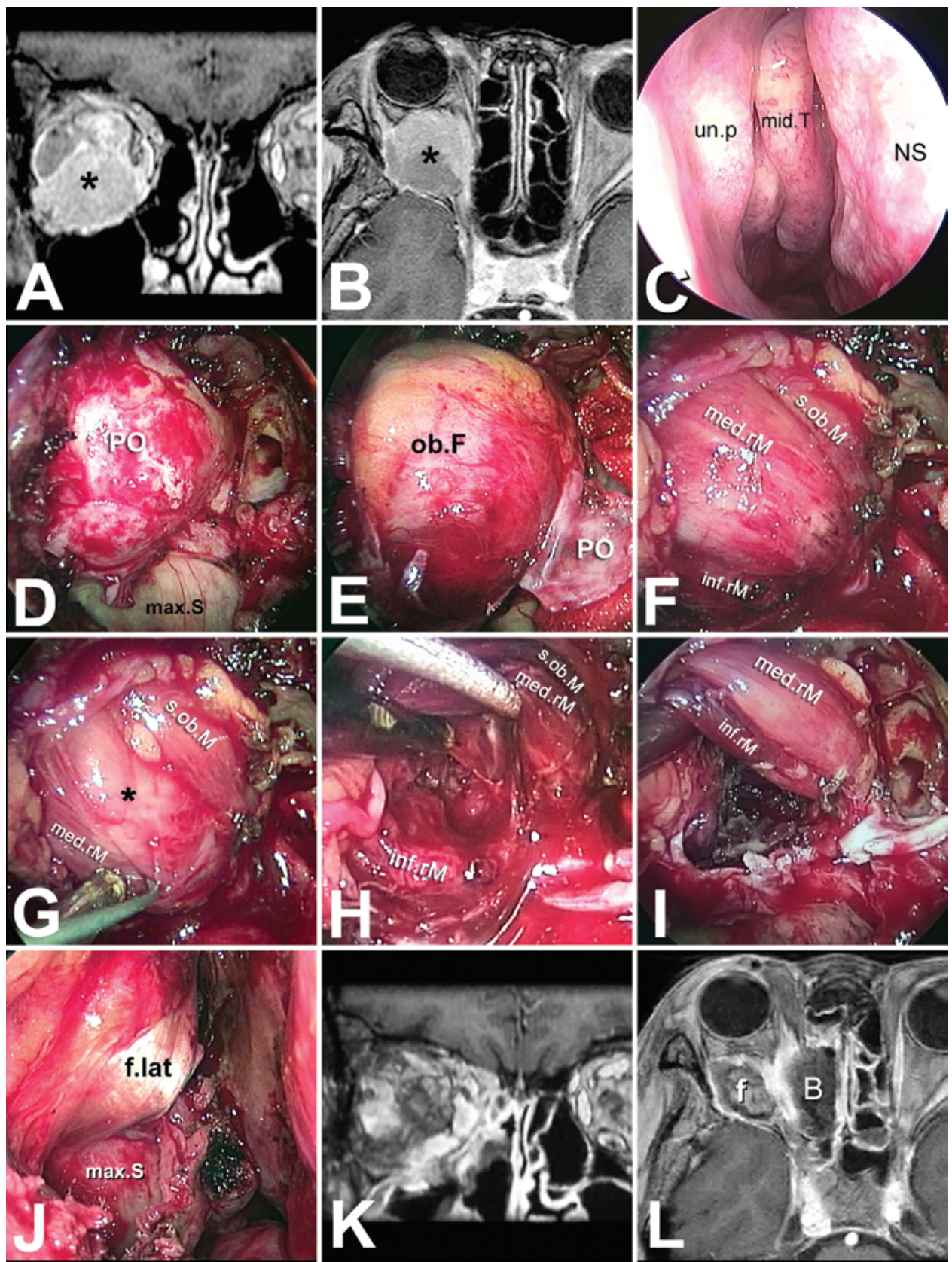

FIG. 5. Case 10. A case of recurrent orbital meningioma. On preoperative Gd-enhanced T1-weighted MR images (A, coronal; B, axial), the heterogeneously enhancing lesion occupies the retrobulbar space and orbital apex in the right orbit (asterisks). Entering the right nasal cavity along the nasal septum (NS), the middle nasal turbinate (mid.T) and uncinate process (un.p) are observed (C). After removal of the middle turbinate and ethmoid air cells with the uncinate process, the maxillary sinus (max.S) and medial orbital wall are exposed. The lamina papyracea is carefully removed, then the periorbita (PO) is widely disclosed (D). On opening the periorbita, the intraorbital fat (ob.F) bulges out (E) and is carefully dissected from underlying muscles and removed. After removal of those redundant tissues, the 3 intraorbital muscles (superior oblique muscle [s.ob.M], medial rectus muscle [med.rM], and inferior rectus muscle [inf.rM]) are disclosed (F). The tumor (asterisk) is easily exposed between those muscles (G), with an approach between the medial and inferior rectus muscles $(\mathrm{H})$ or while medially retracting these 2 muscles (I). After tumor removal, the fascia lata (f.lat) and fat tissue are placed into the cavity. The fascia lata covers the periorbital defect in 2 layers, inside and outside the margin $(\mathrm{J})$ to prevent ocular muscles from bulging out of the defect. After ETS, MR images show that the tumor has been removed and fat tissue ( $f$ ) has been replaced in the cavity (K, coronal; $L$, axial). The inserted fascia $(f)$ is compressed by the balloon $(B)$ from the nasal cavity (in panel L).

periorbita (extraperiosteal lesions) or inside (intraperiosteal lesions).

Extraperiosteal tumors extend from surrounding skull base regions such as the cavernous sinus, sphenoid ridge, or anterior cranial base though the extradural space, or from the paranasal sinus, destroying a medial or upper aspect of the orbital bone. In such cases, the tumor component is usually well demarcated and easily dissected from 

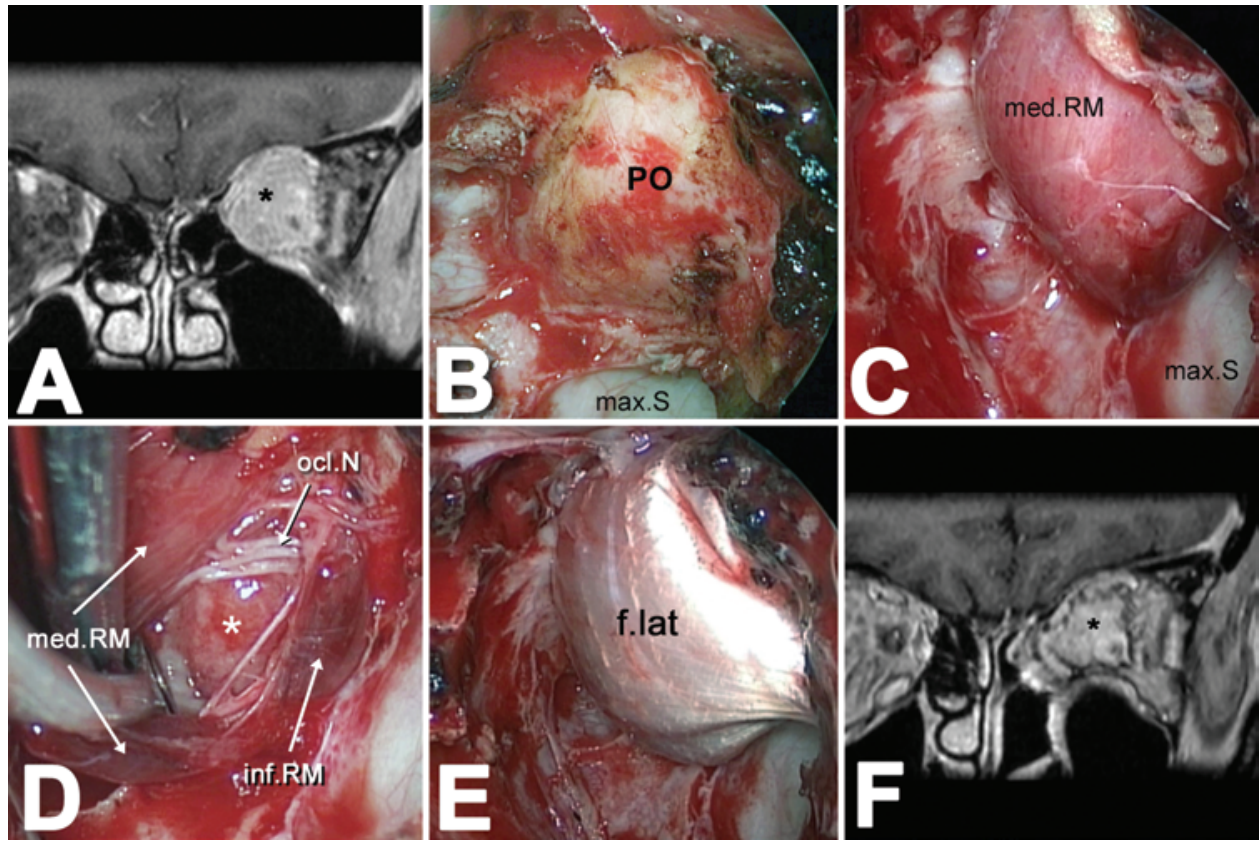

FIG. 6. A case of optic sheath meningioma (Case 11). Preoperative T1-weighted MRI with Gd enhancement (A, coronal section) shows that the left optic nerve is encased in the enhanced lesion (asterisk). After removal of the middle turbinate and ethmoid air cells, the lamina papyracea is carefully removed, then the periorbita (PO) and part of the maxillary sinus (max.S) are exposed (B). On opening the periorbita, the intraorbital fat is carefully dissected, and the medial rectus muscle (med.rM) is disclosed (C). With gentle retraction of the medial rectus muscle, the tumor (asterisk) is exposed and approached between the medial rectus muscle and inferior rectus muscle (inf.rM) through the distal branches of the oculomotor nerve (ocl.N, D). After partial removal of the tumor, the fascia lata (f.lat) covers the periorbital defect $(\mathrm{E})$. Postoperative MRI reveals the residual tumor (asterisk in $\mathrm{F}$, coronal section), but visual symptoms of the patient were markedly improved.

the periorbita or dural membrane of the anterior cranial base. ${ }^{13,18}$ Even if extension is seen to the superior or lateral aspect of the orbit, the tumor is often accessible by using gentle compression of the periorbital, in which all the important anatomical structures are protected. Coagulation and sectioning of the ethmoidal arteries allow further retraction of the periorbita, and gross-total removal is feasible through minimal opening of the lamina papyracea, without an expectation of causing neurological deficit. After resection, reconstruction of the orbit wall is usually unnecessary (Fig. 3). In our series, 9 tumors were located outside the periorbita in the orbital cavity, with 3 tumors showing extension into the superolateral margin. In all 9 cases, gross-total removal was achieved, and only 1 patient experienced mild and transient worsening of facial dysesthesia caused by dissection of the tumor from the infraorbital nerve. Those results indicate that extraperiosteal tumors can be good candidates for ETS, regardless of the lesions' wide extension to the lateral margin.

In contrast, intraperiosteal tumors remain a therapeutic challenge, and undertaking of the transnasal approach should be carefully considered by assessing preoperative radiographic images, operative findings from previous surgeries, presenting symptoms, and the histopathological diagnosis. When visual function of the patient is sufficiently preserved, the course of the optic nerve and its positional relationship to the tumor is verified on MRI. Routes of the central retinal arteries should also be confirmed with cerebral angiography to avoid postoperative retinal ischemia. ${ }^{4,11,15}$ To approach intraconal lesions, we advocate a wide opening of the surgical window through the lamina papyracea and periorbita, regardless of lesion size. After opening the periorbita, the tumor capsule is carefully dissected from the surrounding anatomies such as the medial and inferior rectus muscles or the superior oblique muscles. In response to the reduction of tumor volume during surgery, fat tissue and those muscles gradually prolapse from the surgical window and may hamper the surgical approach to deeper components. With wide orbitotomy, sufficient margins were achieved to effectively retract and displace the extraocular muscles, retracted by a suction tube, with the curved tip in one hand and continued dissection with surgical equipment in the other hand. We could thus stably maintain the approach pathway to all parts of the tumor in the surgical field. Also, in cases of relatively large tumors, gentle compression of the eyeball on the palpebra from outside allows the tumor component behind the eyeball to be easily pushed out from the medial periorbital window, facilitating further dissection and removal of the tumor without any additional incision (Fig. 7). In addition to maintaining a sufficient approach pathway, a wide medial orbitotomy can also more effectively provide optic nerve decompression than expected from reduction of the tumor volume itself, increasing the possibility of improving impaired visual functions. ${ }^{2,20}$

Intraperiosteal tumors, depending on their pathological characteristics, may strongly adhere to the surround- 

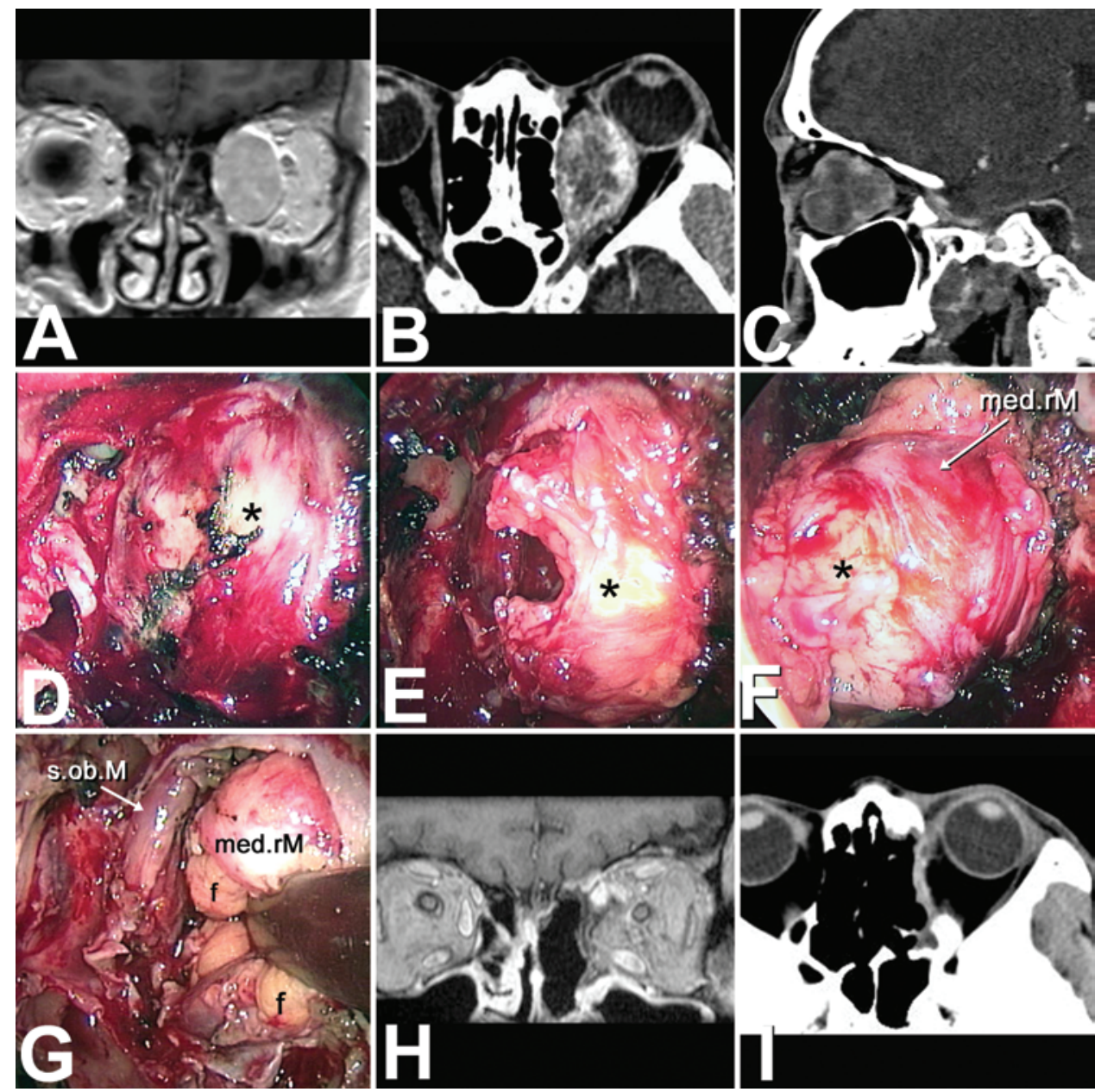

FIG. 7. Case 14. A case of myxofibrosarcoma metastasizing to the medial rectus muscle. The tumor showed rapid enlargement with progressive exophthalmos (A, coronal Gd-enhanced T1-weighted MR image; B, axial CT scan; C, sagittal enhanced CT scan). After wide opening of the periorbital window, the large tumor (asterisk) is exposed (D). The tumor capsule (asterisk) is dissected from the surrounding tissue following internal decompression of the lesion $(\mathbf{E})$. When the eyeball is gently compressed on the upper palpebra from outside, the retrobulbar tumor component is pushed out into the nasal cavity, disclosing that the tumor (asterisk) has invaded into medial rectus muscle (med.rM, F). The tumor is removed with the adjacent part of the medial rectus muscle. The superior oblique muscle (s.ob.M), residual medial rectus muscle, and intraorbital fat tissue (f) are observed (G). After surgery, total resection is confirmed on images ( $\mathrm{H}$, coronal $\mathrm{Gd}$-enhanced T1-weighted MR image; I, axial contrast-enhanced CT).

ing anatomy, such as the optic nerve, extraocular muscle, or peripheral branch of the cranial nerve. Clear dissection thus may not always be possible. The decision in balancing functional preservation and radicality of resection then becomes very difficult, and we should carefully discuss this with the patient before surgery to clarify the priorities of the surgical procedure. In 2 of our patients (Case 10, in the second transnasal surgery, and Case 14), because the tumors showed rapid enlargement and adjuvant therapies were not expected to be efficacious, extirpation of the tumor was considered to be urgent and crucial. As preoperative imaging indicated tumor invasion to the surrounding anatomy, we decided to remove the whole tumor along with adjacent normal structures while abandoning the possibility of functional recovery. The orbit is a relatively restricted area separated from the intracranial space by the several anatomical barriers such as the periorbita, orbital bone, and dural membrane. Even in cases of malignancy, by abandoning the likelihood of functional recovery, we can extend the resection area and possibly improve the radicality and local control rate in a relatively less-invasive fashion.

Our results suggest that the transnasal approach may represent a safe surgical procedure, with minimal invasiveness, even for locally aggressive tumors in the orbit that extend close to the medial and inferior aspects of the orbit. However, depending on the pathology, gross-total resection does not always mean a "cure" of the disease. Because tumor cells occasionally invade adjacent tissues, management of those tissues intraoperatively is important to prevent tumor recurrence. The surgical approach should thus be selected after consideration of those aspects. When the tumor is located in the superior or lateral regions, a transcranial approach will often be most advantageous, facilitating a direct approach to the lesion, requiring minimal retraction of surrounding anatomies and drilling of 


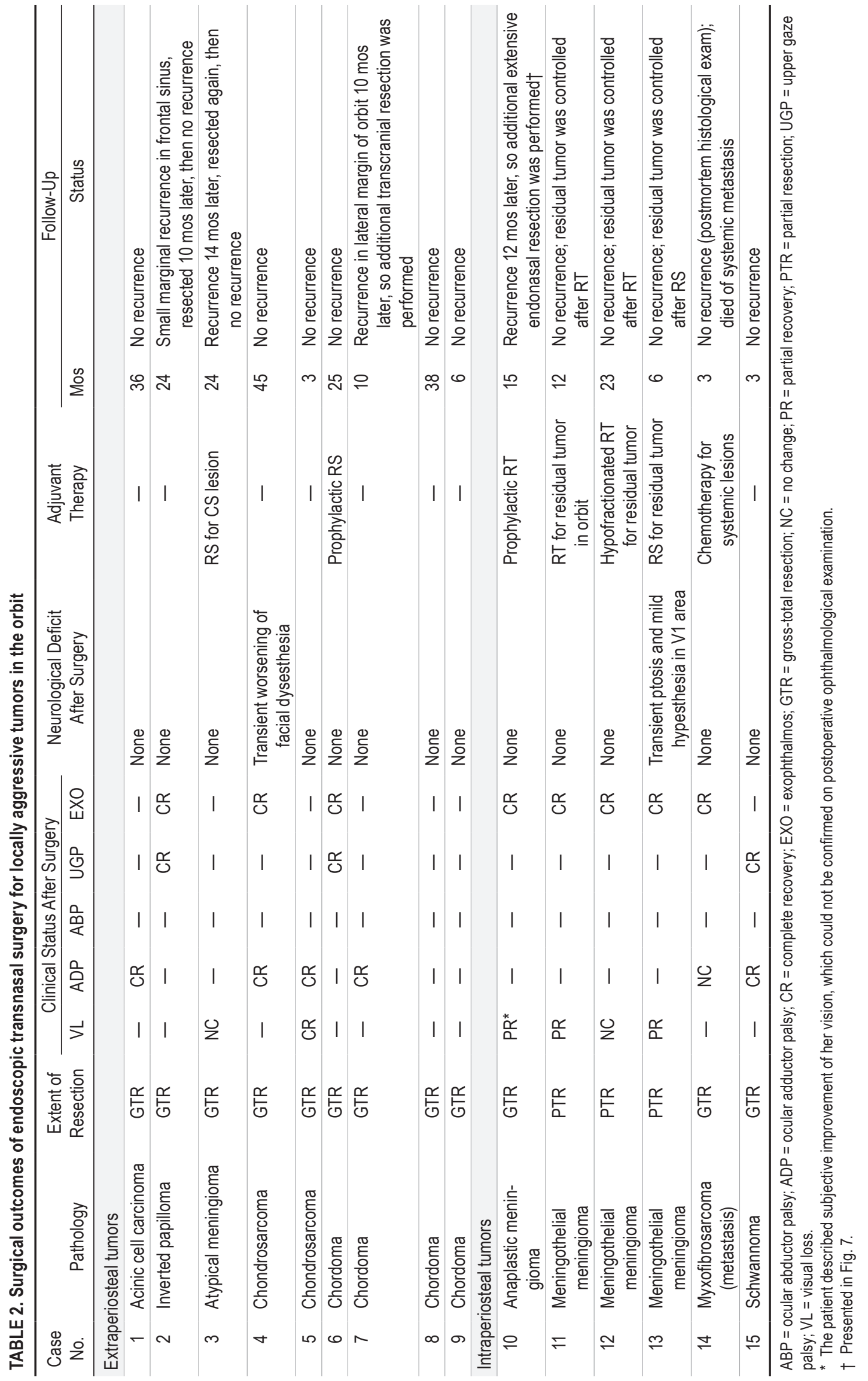




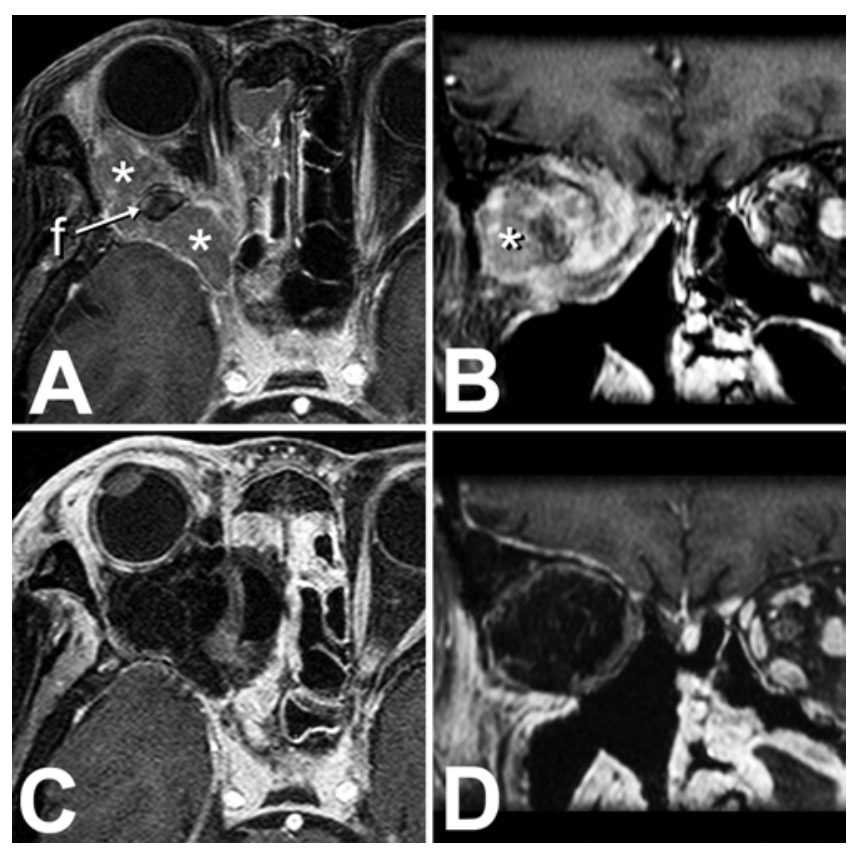

FIG. 8. Case 10. A case of recurrent anaplastic meningioma (second surgery for this patient). Follow-up MR images (T1-weighted Gdenhanced MR images, axial $[A]$ and coronal $[B]$ views) show that the tumor (asterisks) occupies the right orbital space around the fat tissue (f) inserted in the previous surgery. Since her visual function and ocular movements were completely impaired, at surgery, the tumor is removed with all adjacent orbital structures except for the eyeball, and the inner surface of the periorbita is cauterized. Postoperative MR images show total removal of the tumor and replacement of the orbital cavity with fat tissue (T1-weighted MRI with Gd enhancement, axial [C] and coronal [D] views).

adjacent bony structures..$^{1,716}$ Since further accumulation of cases is essential to decisively define the efficacy and safety of an approach, application of transnasal surgery for invasive orbital lesions should be carefully considered for each individual to provide the maximum benefit for patients with those intractable diseases.

\section{Conclusions}

We consider the endoscopic transnasal intraorbital approach as an acceptable, less-invasive alternative for the resection of locally aggressive tumors involving the orbit. Gross-total removal is feasible without neurological complications, regardless of the size and extension of tumors located outside the periorbita. For tumors invading the periorbita, application of the transnasal approach and the decision to balance functional preservation and radicality of resection should be carefully assessed, considering the course of the optic nerve, presenting symptoms, and histology. Wide opening of the periorbital window is advocated to retain a sufficient surgical pathway to facilitate removal of the tumor with minimal neurological deficit, leading to improvement of visual function.

\section{References}

1. Abuzayed B, Kucukyuruk B, Tanriover N, Sanus GZ, Canbaz B, Akar Z, et al: Transcranial superior orbitotomy for the treatment of intraorbital intraconal tumors: surgical technique and long-term results in single institute. Neurosurg $\operatorname{Rev}$ 35:573-582, 2012

2. Acheson JF: Optic nerve disorders: role of canal and nerve sheath decompression surgery. Eye (Lond) 18:1169-1174, 2004

3. Benedičič M, Bošnjak R: Intraoperative monitoring of the visual function using cortical potentials after electrical epidural stimulation of the optic nerve. Acta Neurochir (Wien) 153:1919-1927, 2011

4. Borruat FX, Bogousslavsky J, Uffer S, Klainguti G, Schatz NJ: Orbital infarction syndrome. Ophthalmology 100:562568,1993

5. Cappabianca P, Cavallo LM, de Divitiis E: Endoscopic endonasal transsphenoidal surgery. Neurosurgery 55:933-941, 2004

6. Castelnuovo P, Dallan I, Locatelli D, Battaglia P, Farneti P, Tomazic PV, et al: Endoscopic transnasal intraorbital surgery: our experience with 16 cases. Eur Arch Otorhinolaryngol 269:1929-1935, 2012

7. Ducic Y: Orbitozygomatic resection of meningiomas of the orbit. Laryngoscope 114:164-170, 2004

8. Fernandez-Miranda JC, Morera VA, Snyderman CH, Gardner P: Endoscopic endonasal transclival approach to the jugular tubercle. Neurosurgery 71 (1 Suppl Operative):146-159, 2012

9. Fraser JF, Nyquist GG, Moore N, Anand VK, Schwartz TH: Endoscopic endonasal minimal access approach to the clivus: case series and technical nuances. Neurosurgery 67 (3 Suppl Operative):ons150-ons158, 2010

10. Fukaya C, Katayama Y, Kasai M, Kurihara J, Yamamoto T: Intraoperative electro-oculographic monitoring for skull base surgery. Skull Base Surg 10:11-15, 2000

11. Hayreh SS: Orbital vascular anatomy. Eye (Lond) 20:11301144,2006

12. Jho HD, Carrau RL: Endoscopic endonasal transsphenoidal surgery: experience with 50 patients. J Neurosurg 87:44-51, 1997

13. Karaki M, Akiyama K, Kagawa M, Tamiya T, Mori N: Indications and limitations of endoscopic endonasal orbitotomy for orbital lesion. J Craniofac Surg 23:10931096, 2012

14. Look Hong NJ, Hornicek FJ, Raskin KA, Yoon SS, Szymonifka J, Yeap B, et al: Prognostic factors and outcomes of patients with myxofibrosarcoma. Ann Surg Oncol 20:80-86, 2013

15. Mames RN, Snady-McCoy L, Guy J: Central retinal and posterior ciliary artery occlusion after particle embolization of the external carotid artery system. Ophthalmology 98:527-531, 1991

16. Marcus H, Schwindack C, Santarius T, Mannion R, Kirollos $\mathrm{R}$ : Image-guided resection of spheno-orbital skull-base meningiomas with predominant intraosseous component. Acta Neurochir (Wien) 155:981-988, 2013

17. McKinney KA, Snyderman CH, Carrau RL, Germanwala AV, Prevedello DM, Stefko ST, et al: Seeing the light: endoscopic endonasal intraconal orbital tumor surgery. Otolaryngol Head Neck Surg 143:699-701, 2010

18. Murchison AP, Rosen MR, Evans JJ, Bilyk JR: Endoscopic approach to the orbital apex and periorbital skull base. Laryngoscope 121:463-467, 2011

19. Muscatello L, Seccia V, Caniglia M, Sellari-Franceschini S, Lenzi R: Transnasal endoscopic surgery for selected orbital cavernous hemangiomas: our preliminary experience. Head Neck 35:E218-E220, 2013

20. Pletcher SD, Sindwani R, Metson R: Endoscopic orbital and optic nerve decompression. Otolaryngol Clin North Am 39:943-958, vi, 2006

21. Roth J, Fraser JF, Singh A, Bernardo A, Anand VK, Schwartz 
TH: Surgical approaches to the orbital apex: comparison of endoscopic endonasal and transcranial approaches using a novel 3D endoscope. Orbit 30:43-48, 2011

22. Saeki N, Horiguchi K, Murai H, Hasegawa Y, Hanazawa T, Okamoto Y: Endoscopic endonasal pituitary and skull base surgery. Neurol Med Chir (Tokyo) 50:756-764, 2010

23. Sasaki T, Itakura T, Suzuki K, Kasuya H, Munakata R, Muramatsu $\mathrm{H}$, et al: Intraoperative monitoring of visual evoked potential: introduction of a clinically useful method. J Neurosurg 112:273-284, 2010

24. Schlake HP, Goldbrunner R, Siebert M, Behr R, Roosen $\mathrm{K}$ : Intra-Operative electromyographic monitoring of extraocular motor nerves (Nn. III, VI) in skull base surgery. Acta Neurochir (Wien) 143:251-261, 2001

25. Stippler M, Gardner PA, Snyderman CH, Carrau RL, Prevedello DM, Kassam AB: Endoscopic endonasal approach for clival chordomas. Neurosurgery 64:268-278, 2009

26. Zanation AM, Snyderman CH, Carrau RL, Gardner PA, Prevedello DM, Kassam AB: Endoscopic endonasal surgery for petrous apex lesions. Laryngoscope 119:19-25, 2009

\section{Author Contributions}

Conception and design: Shin, Kondo, Suzukawa, Shojima, Saito. Acquisition of data: Shin, Kondo, Hanakita, Suzukawa, Kin,

Nakagawa. Analysis and interpretation of data: Shin, Kondo, Kin. Drafting the article: Shin. Critically revising the article: Shin, Kondo, Suzukawa, Shojima, Saito. Reviewed submitted version of manuscript: Shin, Kondo, Suzukawa, Saito. Approved the final version of the manuscript on behalf of all authors: Shin. Administrative/technical/material support: Kondo, Hanakita, Suzukawa, Kin, Shojima, Nakagawa, Saito. Study supervision: Saito.

\section{Correspondence}

Masahiro Shin, Department of Neurosurgery, The University of Tokyo Hospital, 7-3-1 Hongo, Bunkyo-ku, Tokyo 133-8655, Japan. email: shin-nsu@h.u-tokyo.ac.jp. 